\title{
Mitochondrial type II NAD(P)H dehydrogenases in fungal cell death
}

\author{
A. Pedro Gonçalves ${ }^{1,2,4}$, Arnaldo Videira ${ }^{1,2,3}$ \\ ${ }^{1}$ ICBAS-Instituto de Ciências Biomédicas de Abel Salazar, Universidade do Porto, Rua de Jorge Viterbo Ferreira 228, 4050-313 Porto, \\ Portugal. \\ ${ }^{2}$ IBMC-Instituto de Biologia Molecular e Celular - Universidade do Porto, Rua do Campo Alegre 823, 4150-180 Porto, Portugal. \\ ${ }^{3}$ Instituto de Investigação e Inovação em Saúde, Universidade do Porto, Portugal. \\ ${ }^{4}$ Current address: Plant and Microbial Biology Department, The University of California, Berkeley, CA 94720, USA. \\ * Corresponding Authors: A. Pedro Gonçalves, E-mail: antoniopedrocardoso@gmail.com; \\ Arnaldo Videira, E-mail: avideira@ibmc.up.pt
}

\begin{abstract}
During aerobic respiration, cells produce energy through oxidative phosphorylation, which includes a specialized group of multi-subunit complexes in the inner mitochondrial membrane known as the electron transport chain. However, this canonical pathway is branched into single polypeptide alternative routes in some fungi, plants, protists and bacteria. They confer metabolic plasticity, allowing cells to adapt to different environmental conditions and stresses. Type II NAD(P)H dehydrogenases (also called alternative $\mathrm{NAD}(\mathrm{P}) \mathrm{H}$ dehydrogenases) are non-proton pumping enzymes that bypass complex I. Recent evidence points to the involvement of fungal alternative $\mathrm{NAD}(\mathrm{P}) \mathrm{H}$ dehydrogenases in the process of programmed cell death, in addition to their action as overflow systems upon oxidative stress. Consistent with this, alternative $\mathrm{NAD}(\mathrm{P}) \mathrm{H}$ dehydrogenases are phylogenetically related to cell death - promoting proteins of the apoptosis-inducing factor (AIF)-family.
\end{abstract}

\author{
doi: 10.15698/mic2015.03.192 \\ Received originally: 15.12.2014; \\ in revised form: 20.02.2015, \\ Accepted 21.02.2015 \\ Published 02.03.2015.
}

Keywords: alternative $N A D(P) H$ dehydrogenases, fungi, programmed cell death, ROS.

\section{INTRODUCTION}

Mitochondria (from the Greek mitos and chondros, meaning "thread" and "granule", respectively) are the dynamos of the eukaryotic cell due to their major role in energy production under aerobic conditions. They are double membrane organelles: the protein-rich core of the organelle is known as the matrix, whereas the inner and outer mitochondrial membranes (IMM and OMM, respectively) delimitate the intermembrane space. The inner membrane forms a series of invaginations designated as cristae. Mitochondria take up pyruvate formed during the first stage of carbon metabolism (glycolysis) and fatty acids, and convert them into energy [1]. The respiratory chain in the IMM is composed of the multi-subunit enzymatic complexes (complex I, II, III and IV), together with ubiquinone (coenzyme Q) and cytochrome $c$ (Fig. 1). These complexes possess a number of protein-associated prosthetic groups flavin mononucleotide (FMN), flavin adenine dinucleotide (FAD), iron-sulfur clusters (FeS), iron and copper ions and heme - that transport electrons. Ubiquinone and cytochrome $c$ transfer electrons between complexes. The electrochemical gradient that triggers the rotation of the ATP synthase (complex V), which leads to the formation of ATP from the phosphorylation of ADP [2, 3], is generated by the proton-pumping activity of (i) complex I (NADH:ubiquinone oxidoreductase), which uses $\mathrm{NADH}$ as a source of electrons, transferring them to ubiquinone via FMN and a series of iron-sulfur clusters, (ii) complex III (ubiquinol cytochrome $c$ reductase), which transfers electrons from the reduced ubiquinone or ubiquinol to cytochrome $c$, and (iii) complex IV (cytochrome $c$ oxidase), which catalyses electron transfer to molecular oxygen and reduces it to water. Complex II (succinate dehydrogenase) transfers electrons from succinate to ubiquinone, providing an alternative electron entry point into the respiratory chain without proton pumping. Apart from the generation of energy, mitochondria are involved in several other cellular processes, like the biogenesis of iron-sulphur clusters, $\mathrm{Ca}^{2+}$ storage, intermediary metabolism, coenzyme biosynthesis and cell death [1].

\section{BRANCHED RESPIRATORY SYSTEMS IN FUNGI}

In some fungi, plants, protists and bacteria, the electron transport chain is branched into single polypeptide alterna- 

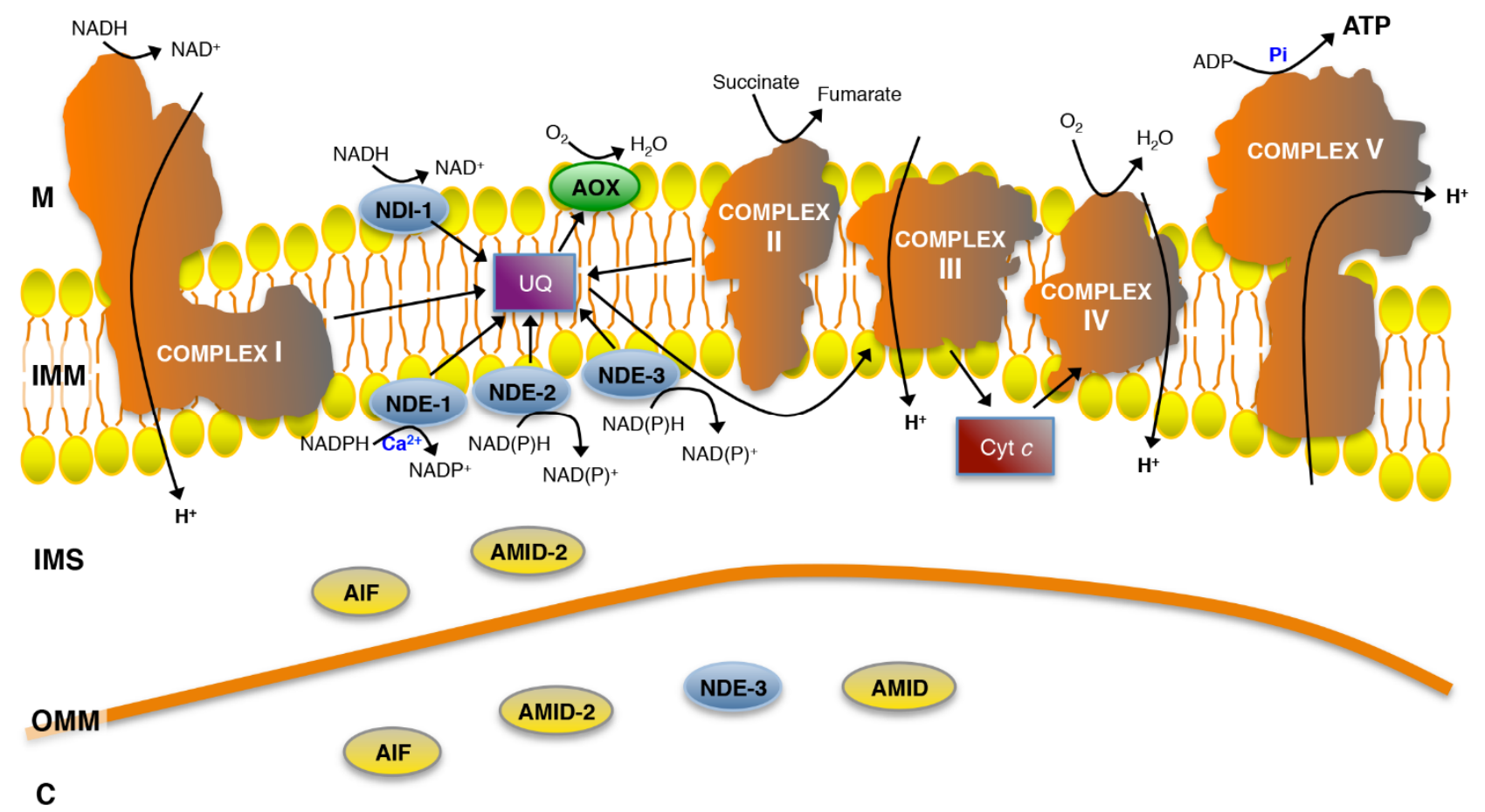

FIGURE 1: Representation of the mitochondrial respiratory chain, alternative NAD(P)H dehydrogenases, alternative oxidase systems and AIF-family proteins of $\boldsymbol{N}$. crassa. M: mitochondrial matrix; IMM: mitochondrial inner membrane; IMS: intermembrane space; OMM: mitochondrial outer membrane; C: cytosol; UQ: ubiquinone; Cyt c: cytochrome $c$.

tive systems with no proton translocation activity that bypass the canonical pathway. The cyanide-resistant alternative oxidase (AOX) constitutes a well-established bypass of complexes III and IV, whereas type II NAD(P)H dehydrogenases (or alternative $\mathrm{NAD}(\mathrm{P}) \mathrm{H}$ dehydrogenases) bypass complex I $[4,5]$. Alternative $\mathrm{NAD}(\mathrm{P}) \mathrm{H}$ dehydrogenases are particularly important not only because they oxidize $\mathrm{NAD}(\mathrm{P}) \mathrm{H}$ and reduce quinones but also because they serve as entry points for electrons into the respiratory chain $[6$, 7]. Their importance is firmly demonstrated in Saccharomyces cerevisiae, where complex I is absent [8] and type II $\mathrm{NAD}(\mathrm{P}) \mathrm{H}$ dehydrogenases are the only existing $\mathrm{NAD}(\mathrm{P}) \mathrm{H}$ oxidases $[9,10]$. These enzymes are flavoproteins resistant to classical complex I inhibitors, such as rotenone or piericidin $A$, and there is no selective and reliable drug to block them so far, although inhibition is feasible with diphenyleneiodonium $[11,12,13]$. Alternative $\mathrm{NAD}(\mathrm{P}) \mathrm{H}$ dehydrogenases usually, but not always, contain FAD as the sole prosthetic group [4, 5, 7]. Recently, an in silico approach identified putative alternative $\mathrm{NAD}(\mathrm{P}) \mathrm{H}$ dehydrogenases in a few metazoan organisms, but a functional verification is still missing [14].

In addition to complex I, our group characterized four alternative rotenone-insensitive $\mathrm{NAD}(\mathrm{P}) \mathrm{H}$ dehydrogenases in Neurospora crassa (Fig. 1 and Table 1) [6, 7]. They are associated with the inner mitochondrial membrane, but while one of them, NDI-1 [15], is localized at the matrix side of the membrane, the other three, NDE-1 $[16,17]$,
NDE-2 [18] and NDE-3 [19], are facing the intermembrane space. Interestingly, NDE-3 was also found in the cytosol [19].

Plants contain even more type II NAD(P)H dehydrogenases than fungi. Seven of these enzymes have been identified in Arabidopsis thaliana: three external (NDB1, NDB2 and NDB4), three internal (NDA1, NDA2 and NDC1) and one uncharacterized (NDB3) [20-22]. Motifs in the $\mathrm{N}^{\prime}$ terminal portion of the proteins appear to determine mitochondrial import and their localization to either side of the inner membrane [23]. Interestingly, dual targeting to mitochondria and chloroplasts or peroxisomes was claimed in some cases, although its functional relevance is unknown [24].

Alternative $\mathrm{NAD}(\mathrm{P}) \mathrm{H}$ dehydrogenases may be organized in supramolecular entities, similarly to the respiratory chain supercomplexes. There is evidence showing that in yeast these enzymes form a complex with a glycerol-3phosphate dehydrogenase, two L-lactate-dehydrogenases, a few enzymes from the tricarboxylic acid cycle, two probable flavoproteins and an acetaldehyde dehydrogenase [25]. In addition, the Yarrowia lipolytica alternative external NADH dehydrogenase and complex IV are associated, particularly in high energy-requiring, logarithmic-growth phase cells $[26,27]$. Current literature suggests that the formation of supercomplexes, that include $\mathrm{NAD}(\mathrm{P}) \mathrm{H}$ dehydrogenases, might be related with electron channelling $[25,27]$. In $N$. crassa, there is no evidence for the for- 
TABLE 1. Main features of $N$. crassa alternative NAD(P)H dehydrogenases.

\begin{tabular}{lllllr}
\hline Protein & Topology & Substrate specificity & $\mathrm{Ca}^{2+}$ & $\mathbf{p H}$ & Reference(s) \\
\hline NDE-1 & External & Cytosolic NADPH & $\mathrm{Ca}^{2+}$-dependent & Physiological pH & {$[16,17]$} \\
\hline NDE-2 & External & Cytosolic NADH and NADPH & - & $\begin{array}{l}\text { NADH throughout the } \\
\text { range and NADPH at acidic } \mathrm{pH}\end{array}$ & {$[18]$} \\
\hline NDE-3 & External & Cytosolic NADH and NADPH & - & $\mathrm{NA}$ & {$[19]$} \\
\hline NDI-1 & Internal & Matrix NADH & $\mathrm{Ca}^{2+}$-stimulated? ${ }^{\mathrm{a}}$ & $\mathrm{NA}$ & {$[15,18]$} \\
\hline
\end{tabular}

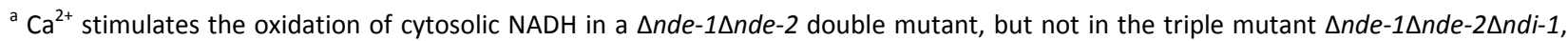
indicating that NDI-1 may be stimulated by $\mathrm{Ca}^{2+}[18]$. NA: not assessed.

mation of supercomplexes containing alternative $\mathrm{NAD}(\mathrm{P}) \mathrm{H}$ dehydrogenases [28], but observations point to some kind of interaction between NDE-2 and complex I [18]. N. crassa NDE-1 stands out because of its unique NADPH selectivity and regulation by $\mathrm{pH}$ and $\mathrm{Ca}^{2+}$ [17], the latter feature likely related to the presence of a conserved $\mathrm{Ca}^{2+}$-binding domain [16]. In plants, the external NDB1 oxidizes NADPH in a $\mathrm{Ca}^{2+}$-dependent manner while NDB2 is a NADH dehydrogenase stimulated by $\mathrm{Ca}^{2+}[22,29]$.

The physiological role of alternative $\mathrm{NAD}(\mathrm{P}) \mathrm{H}$ dehydrogenases is still somehow controversial, although it is fairly well established that they confer metabolic plasticity allowing cells to adapt to different environmental and stress conditions. They may act as overflow systems keeping cytosolic and mitochondrial reducing equivalents (NADH, NADPH) at physiological levels, thus avoiding potential tricarboxylic cycle repression by elevated NADH levels and excessive levels of reactive oxygen species (ROS) $[4,5,7$, 30]. Heterologous expression of $\mathrm{Ndi1}$ from yeast was shown to reduce mammalian complex I-mediated ROS generation [31]. In contrast, S. cerevisiae alternative $\mathrm{NADH}$ dehydrogenases have been proposed as potential sources of superoxide radicals by other studies [32-34].

In N. crassa, expression of alternative $\mathrm{NAD}(\mathrm{P}) \mathrm{H}$ dehydrogenases genes greatly depends on the growth phase $[15,18,19,35]$. It is not possible to obtain viable double mutants between NDE-2 and complex I mutants that lack a functional enzyme, suggesting that NDE-2 and complex I interact in a yet unidentified pathway [18].

Humans do not possess alternative $\mathrm{NAD}(\mathrm{P}) \mathrm{H}$ dehydrogenases, but enzymes from other organisms have potential to be used in gene-based therapies. Heterologous expression of the yeast Ndi1 restores respiration in complex Ideficient human cells [36] and was also shown to be protective in in vivo models of Parkinson's disease [37, 38], Leber's hereditary optic neuropathy [39] and breast cancer [40].

Because mammals lack alternative $\mathrm{NAD}(\mathrm{P}) \mathrm{H}$ dehydrogenases, these enzymes are good candidate targets for human therapy in cases of fungal infection. The crystal structure of yeast $\mathrm{Ndi1}$ has been recently solved and will allow a better understanding of the regulatory mechanisms of type II $\mathrm{NAD}(\mathrm{P}) \mathrm{H}$ dehydrogenases and likely lead to an evaluation of their potential as therapeutical agents or targets [41, 42].

\section{ALTERNATIVE NAD(P)H DEHYDROGENASES AS MEDIATORS OF PROGRAMMED CELL DEATH}

Several reports point to a role of fungal alternative $\mathrm{NAD}(\mathrm{P}) \mathrm{H}$ dehydrogenases in cell death. In $\mathrm{S}$. cerevisiae, overexpression of the internal Ndi1 (proposed as the yeast homologue of the human apoptosis-inducing factorhomologous mitochondrion-associated inducer of death or AMID), but not of the external Nde1, leads to ROSmediated apoptosis-like cell death, particularly in glucoserich media. The authors showed that the disruption of both of these NADH dehydrogenases results in lower ROS production and increased chronological life span accompanied by reduced fitness [34].

More recently, yeast Ndi1 was also shown to be involved in cell death induced by different stimuli like hydrogen peroxide, acetic acid and manganese ions, independently of its oxidoreductase activity [43]. During the execution of manganese ion-induced cell death, a $\mathrm{N}^{\prime}$ terminal portion of $\mathrm{Ndi1}$ is cleaved and the protein translocates to the cytoplasm. However, in sharp contrast, it was reported that overexpression of yeast Ndi1 in human cell lines prevents rotenone- and paraquat-induced cell death $[44,45]$.

Thus, the specific role of alternative $N A D(P) H$ dehydrogenases in the protection or enhancement of ROS production is still uncertain [27, 31, 32, 45-48]. Although speculative, it is possible that the discrepant findings observed in human and yeast cells overexpressing Ndi1 result from the fact that on the one hand, human cells do not possess these enzymes, and one the other hand, yeast cells harbor additional NAD(P)H dehydrogenases. In both cases, this suggests that alternative $\mathrm{NAD}(\mathrm{P}) \mathrm{H}$ dehydrogenases may interact with each other or respond to overexpression or downregulation of other members of the family. In fact, compensatory mechanisms of gene expression have been demonstrated in $N$. crassa strains lacking one or more $\mathrm{NAD}(\mathrm{P}) \mathrm{H}$ dehydrogenases (see below).

In N. crassa, a double mutant devoid of NDE-1 and NDE-2 displays lower ROS accumulation, increased catalase activity and resistance to paraquat [46]. Particularly, NDE-2 
appears to be engaged in mitochondrial ROS generation. In $A$. nidulans, the expression of genes encoding $\mathrm{NAD}(\mathrm{P}) \mathrm{H}$ dehydrogenases are induced upon exposure to different cell death stimuli, especially farnesol [49]. Moreover, while the overexpression of $\mathrm{NdiA}$ augments the resistance to farnesol, the deletion of NdeA results in hypersensitivity to the drug. The latter is likely due to increased accumulation of ROS in the presence of farnesol [49]. In N. crassa, disruption of nde-1 leads to increased susceptibility to staurosporine, associated with higher ROS accumulation and altered intracellular $\mathrm{Ca}^{2+}$ dynamics (Gonçalves AP, Cordeiro JM, Monteiro J, Lucchi C, Correia-de-Sá P, Videira A, unpublished data). In addition, a yeast NDE1 deletion strain is more resistant to artemisinin and dimeric naphthoquinones [50, 51]. Despite the aforementioned controversy around the role of $\mathrm{NAD}(\mathrm{P}) \mathrm{H}$ dehydrogenases during cell death, a current view is that these enzymes seem to be activated in different model organisms in conditions of highly reducing cellular environment, diverging electron transfer from the canonical respiratory chain pathway and thus avoiding system overflow and deleterious ROS production [27, 47].

Notably, alternative $\mathrm{NAD}(\mathrm{P}) \mathrm{H}$ dehydrogenases are protein homologues of apoptosis-inducing factor (AIF)-family members, namely the well established cell death executioners AIF and AMID (Fig. 1). AIF-family members have been described as oxidoreductases [52, 53], but disruption of AIF or AMID does not affect complex I activity, nor does the supramolecular organization of the respiratory chain in N. crassa [35]. In this fungus, AIF was found both in mitochondrial and cytosolic extracts [35], while, for comparison, two AIFs localized in the mitochondria and in the cytoplasm, respectively, have been reported in Podospora anserina [54]. In N. crassa, AMID was found exclusively in the cytosol whereas a third member of this family, AMID-2, was found both in the mitochondria and in the cytosol but was only observed when AMID was absent, suggesting overlapping functions [35]. A genome-wide association study on a wild population of $N$. crassa showed a genetic interaction between amid-2 and czt-1, a transcription factor that controls cell death and drug resistance [55].

In $N$. crassa, analysis of gene expression profiles of these families of genes in simple or multiple deletion strains for alternative $\mathrm{NAD}(\mathrm{P}) \mathrm{H}$ dehydrogenases showed the occurrence of compensatory mechanisms [19, 35, 46]. For instance, $n d i-1$ is upregulated in a $\Delta n d e-1 \Delta n d e-2$ and in a $\Delta$ nde-2 strain, amid and amid-2 are upregulated in a triple $\Delta n d i-1 \Delta n d e-1 \Delta n d e-2$ mutant, nde-1 is upregulated in $\Delta n d e-3$ cells and $n d e-2$ is upregulated in $\Delta n d i-1, \Delta n d e-1$ and $\Delta n d e-3$ single mutants. The functional meaning of this compensation in gene expression is currently unknown, but there is evidence in yeast that mitochondrial dysfunction leads to an alteration in gene expression through retrograde signaling in order to reduce the impact of this dysfunction on cellular fitness [56]. Interestingly, a phylogenetic analysis showed that $N$. crassa NDE-3 clusters with AIF-like proteins rather than with the other NAD(P)H dehydrogenases [35], further suggesting a close relationship between these proteins.

Accumulating evidence clearly relates alternative $\mathrm{NAD}(\mathrm{P}) \mathrm{H}$ dehydrogenases to intracellular cell death routes. However, further studies are needed to better understand the mechanisms underlying their involvement in the cellular responses to cell death stimuli.

\section{ACKNOWLEDGEMENTS}

A.P.G. was recipient of a fellowship from Fundação Calouste Gulbenkian (104210) and a fellowship from COMPETE - Programa Operacional Factores de Competitividade (PEst-LA051301-BTI).

\section{CONFLICT OF INTEREST}

The authors declare no conflict of interest.

\section{COPYRIGHT}

(C) 2015 Gonçalves and Videira. This is an open-access article released under the terms of the Creative Commons Attribution (CC BY) license, which allows the unrestricted use, distribution, and reproduction in any medium, provided the original author and source are acknowledged.

Please cite this article as: A. Pedro Gonçalves, Arnaldo Videira (2015). Mitochondrial type II NAD(P)H dehydrogenases in fungal cell death. Microbial Cell 2(3): 68-73. doi: 10.15698/mic2015.03.192

5. Rasmusson AG, Geisler DA, Moller IM (2008). The multiplicity of dehydrogenases in the electron transport chain of plant mitochondria. Mitochondrion 8(1): 47-60.

6. Videira A, Duarte $M$ (2001). On complex $I$ and other $\mathrm{NADH}$ :ubiquinone reductases of Neurospora crassa mitochondria. J Bioenerg Biomembr 33(3): 197-203.

7. Videira A, Duarte $M$ (2002). From $N A D H$ to ubiquinone in Neurospora mitochondria. Biochim Biophys Acta 1555(1-3): 187-191.

8. Buschges R, Bahrenberg G, Zimmermann M, Wolf K (1994). NADH: ubiquinone oxidoreductase in obligate aerobic yeasts. Yeast 10(4): 475-479.
4. Melo AM, Bandeiras TM, Teixeira M (2004). New insights into type II NAD(P)H:quinone oxidoreductases. Microbiol Mol Biol Rev 68(4): 603-616.
3. Lenaz G, Genova ML (2010). Structure and organization of mitochondrial respiratory complexes: a new understanding of an old subject. Antioxid Redox Signal 12(8): 961-1008. 
9. De Vries S, Van Witzenburg R, Grivell LA, Marres CA (1992). Primary structure and import pathway of the rotenone-insensitive NADHubiquinone oxidoreductase of mitochondria from Saccharomyces cerevisiae. Eur J Biochem 203(3): 587-592.

10. Luttik MA, Overkamp KM, Kotter P, de Vries S, van Dijken JP, Pronk JT (1998). The Saccharomyces cerevisiae NDE1 and NDE2 genes encode separate mitochondrial NADH dehydrogenases catalyzing the oxidation of cytosolic NADH. J Biol Chem 273(38): 24529-24534.

11. Dong CK, Patel V, Yang JC, Dvorin JD, Duraisingh MT, Clardy J, Wirth DF (2009). Type II NADH dehydrogenase of the respiratory chain of Plasmodium falciparum and its inhibitors. Bioorg Med Chem Lett 19(3): 972-975.

12. Roberts TH, Fredlund KM, Moller IM (1995). Direct evidence for the presence of two external $\mathrm{NAD}(\mathrm{P}) \mathrm{H}$ dehydrogenases coupled to the electron transport chain in plant mitochondria. FEBS Lett 373(3): 307309

13. Agius SC, Bykova NV, Igamberdiev AU, Moller IM (1998). The internal rotenone-insensitive NADPH dehydrogenase contributes to malate oxidation by potato tuber and pea leaf mitochondria. Physiol Plant 104(329-336.

14. Matus-Ortega MG, Salmeron-Santiago KG, Flores-Herrera O, Guerra-Sanchez G, Martinez F, Rendon JL, Pardo JP (2011). The alternative NADH dehydrogenase is present in mitochondria of some animal taxa. Comp Biochem Physiol Part D Genomics Proteomics 6(3): 256-263.

15. Duarte $M$, Peters $M$, Schulte U, Videira A (2003). The internal alternative $\mathrm{NADH}$ dehydrogenase of Neurospora crassa mitochondria. Biochem J 371(Pt 3): 1005-1011.

16. Melo AM, Duarte M, Videira A (1999). Primary structure and characterisation of a $64 \mathrm{kDa} \mathrm{NADH}$ dehydrogenase from the inner membrane of Neurospora crassa mitochondria. Biochim Biophys Acta 1412(3): 282-287.

17. Melo AM, Duarte M, Moller IM, Prokisch H, Dolan PL, Pinto L, Nelson MA, Videira A (2001). The external calcium-dependent NADPH dehydrogenase from Neurospora crassa mitochondria. J Biol Chem 276(6): 3947-3951.

18. Carneiro $P$, Duarte $M$, Videira A (2004). The main external alternative $\mathrm{NAD}(\mathrm{P}) \mathrm{H}$ dehydrogenase of Neurospora crassa mitochondria. Biochim Biophys Acta 1608(1): 45-52.

19. Carneiro $P$, Duarte $M$, Videira $A$ (2007). The external alternative $\mathrm{NAD}(\mathrm{P}) \mathrm{H}$ dehydrogenase NDE3 is localized both in the mitochondria and in the cytoplasm of Neurospora crassa. J Mol Biol 368(4): 11141121.

20. Elhafez D, Murcha MW, Clifton R, Soole KL, Day DA, Whelan J (2006). Characterization of mitochondrial alternative $\mathrm{NAD}(\mathrm{P}) \mathrm{H}$ dehydrogenases in Arabidopsis: intraorganelle location and expression. Plant Cell Physiol 47(1): 43-54.

21. Michalecka AM, Svensson AS, Johansson FI, Agius SC, Johanson U, Brennicke A, Binder S, Rasmusson AG (2003). Arabidopsis genes encoding mitochondrial type II NAD(P)H dehydrogenases have different evolutionary origin and show distinct responses to light. Plant Physiol 133(2): 642-652.

22. Geisler DA, Broselid C, Hederstedt L, Rasmusson AG (2007). Ca2+binding and $\mathrm{Ca} 2+-$ independent respiratory $\mathrm{NADH}$ and $\mathrm{NADPH}$ dehydrogenases of Arabidopsis thaliana. J Biol Chem 282(39): 2845528464.

23. Rasmusson AG, Svensson AS, Knoop V, Grohmann L, Brennicke A (1999). Homologues of yeast and bacterial rotenone-insensitive NADH dehydrogenases in higher eukaryotes: two enzymes are present in potato mitochondria. Plant J 20(1): 79-87.
24. Carrie C, Murcha MW, Kuehn K, Duncan O, Barthet $M$, Smith PM, Eubel H, Meyer E, Day DA, Millar AH, Whelan J (2008). Type II NAD(P)H dehydrogenases are targeted to mitochondria and chloroplasts or peroxisomes in Arabidopsis thaliana. FEBS Lett 582(20): 3073-3079.

25. Grandier-Vazeille X, Bathany K, Chaignepain S, Camougrand N, Manon S, Schmitter JM (2001). Yeast mitochondrial dehydrogenases are associated in a supramolecular complex. Biochemistry 40(33): 9758-9769.

26. Guerrero-Castillo $S$, Vazquez-Acevedo $M$, Gonzalez-Halphen $D$, Uribe-Carvajal S (2009). In Yarrowia lipolytica mitochondria, the alternative $\mathrm{NADH}$ dehydrogenase interacts specifically with the cytochrome complexes of the classic respiratory pathway. Biochim Biophys Acta 1787(2): 75-85.

27. Guerrero-Castillo S, Cabrera-Orefice A, Vazquez-Acevedo $M$, Gonzalez-Halphen D, Uribe-Carvajal S (2012). During the stationary growth phase, Yarrowia lipolytica prevents the overproduction of reactive oxygen species by activating an uncoupled mitochondrial respiratory pathway. Biochim Biophys Acta 1817(2): 353-362.

28. Marques I, Dencher NA, Videira A, Krause F (2007). Supramolecular organization of the respiratory chain in Neurospora crassa mitochondria. Eukaryot Cell 6(12): 2391-2405.

29. Michalecka AM, Agius SC, Moller IM, Rasmusson AG (2004). Identification of a mitochondrial external NADPH dehydrogenase by overexpression in transgenic Nicotiana sylvestris. Plant J 37(3): 415425.

30. Rasmusson AG, Wallstrom SV (2010). Involvement of mitochondria in the control of plant cell $\mathrm{NAD}(\mathrm{P}) \mathrm{H}$ reduction levels. Biochem Soc Trans 38(2): 661-666.

31. Seo BB, Marella M, Yagi T, Matsuno-Yagi A (2006). The single subunit $\mathrm{NADH}$ dehydrogenase reduces generation of reactive oxygen species from complex I. FEBS Lett 580(26): 6105-6108.

32. Fang J, Beattie DS (2003). External alternative NADH dehydrogenase of Saccharomyces cerevisiae: a potential source of superoxide. Free Radic Biol Med 34(4): 478-488.

33. Davidson JF, Schiestl RH (2001). Mitochondrial respiratory electron carriers are involved in oxidative stress during heat stress in Saccharomyces cerevisiae. Mol Cell Biol 21(24): 8483-8489.

34. Li W, Sun L, Liang Q, Wang J, Mo W, Zhou B (2006). Yeast AMID homologue Ndi1p displays respiration-restricted apoptotic activity and is involved in chronological aging. Mol Biol Cell 17(4): 1802-1811.

35. Carneiro P, Duarte M, Videira A (2012). Characterization of apoptosis-related oxidoreductases from Neurospora crassa. PLoS One 7(3): e34270.

36. Seo BB, Kitajima-Ihara T, Chan EK, Scheffler IE, Matsuno-Yagi A, Yagi T (1998). Molecular remedy of complex I defects: rotenoneinsensitive internal $\mathrm{NADH}$-quinone oxidoreductase of Saccharomyces cerevisiae mitochondria restores the NADH oxidase activity of complex I-deficient mammalian cells. Proc Natl Acad Sci U S A 95(16): 9167-9171.

37. Seo BB, Nakamaru-Ogiso E, Flotte TR, Matsuno-Yagi A, Yagi T (2006). In vivo complementation of complex I by the yeast Ndi1 enzyme. Possible application for treatment of Parkinson disease. J Biol Chem 281(20): 14250-14255.

38. Marella M, Seo BB, Nakamaru-Ogiso E, Greenamyre JT, MatsunoYagi A, Yagi $T$ (2008). Protection by the NDI1 gene against neurodegeneration in a rotenone rat model of Parkinson's disease. PLoS One 3(1): e1433.

39. Marella M, Seo BB, Thomas BB, Matsuno-Yagi A, Yagi T (2010). Successful amelioration of mitochondrial optic neuropathy using the yeast NDI1 gene in a rat animal model. PLoS One 5(7): e11472. 
40. Santidrian AF, Matsuno-Yagi A, Ritland M, Seo BB, LeBoeuf SE, Gay L, Yagi T, Felding-Habermann B (2013). Mitochondrial complex activity and $\mathrm{NAD+} / \mathrm{NADH}$ balance regulate breast cancer progression. J Clin Invest 123(3): 1068-1081

41. Feng Y, Li W, Li J, Wang J, Ge J, Xu D, Liu Y, Wu K, Zeng Q, Wu JW, Tian C, Zhou B, Yang M (2012). Structural insight into the type-II mitochondrial NADH dehydrogenases. Nature 491(7424): 478-482.

42. Iwata $M$, Lee $Y$, Yamashita T, Yagi T, Iwata S, Cameron AD, Maher MJ (2012). The structure of the yeast NADH dehydrogenase (Ndi1) reveals overlapping binding sites for water- and lipid-soluble substrates. Proc Natl Acad Sci U S A 109(38): 15247-15252.

43. Cui Y, Zhao S, Wu Z, Dai P, Zhou B (2012). Mitochondrial release of the NADH dehydrogenase Ndi1 induces apoptosis in yeast. Mol Biol Cell 23(22): 4373-4382.

44. Marella M, Seo BB, Matsuno-Yagi A, Yagi T (2007). Mechanism of cell death caused by complex I defects in a rat dopaminergic cell line. J Biol Chem 282(33): 24146-24156.

45. Park JS, Li YF, Bai Y (2007). Yeast NDI1 improves oxidative phosphorylation capacity and increases protection against oxidative stress and cell death in cells carrying a Leber's hereditary optic neuropathy mutation. Biochim Biophys Acta 1772(5): 533-542.

46. Carneiro $P$, Duarte $M$, Videira A (2012). Disruption of alternative $\mathrm{NAD}(\mathrm{P}) \mathrm{H}$ dehydrogenases leads to decreased mitochondrial ROS in Neurospora crassa. Free Radic Biol Med 52(2): 402-409.

47. Voulgaris I, O'Donnell A, Harvey LM, McNeil B (2012). Inactivating alternative $\mathrm{NADH}$ dehydrogenases: enhancing fungal bioprocesses by improving growth and biomass yield? Sci Rep 2(322).

48. O'Donnell A, Harvey LM, McNeil B (2011). The roles of the alternative $\mathrm{NADH}$ dehydrogenases during oxidative stress in cultures of the filamentous fungus Aspergillus niger. Fungal Biol 115(4-5): 359369.
49. Dinamarco TM, Pimentel Bde C, Savoldi M, Malavazi I, Soriani FM, Uyemura SA, Ludovico P, Goldman MH, Goldman GH (2010). The roles played by Aspergillus nidulans apoptosis-inducing factor (AIF)-like mitochondrial oxidoreductase (AifA) and NADH-ubiquinone oxidoreductases (NdeA-B and $\mathrm{NdiA}$ ) in farnesol resistance. Fungal Genet Biol 47(12): 1055-1069.

50. Li W, Mo W, Shen D, Sun L, Wang J, Lu S, Gitschier JM, Zhou B (2005). Yeast model uncovers dual roles of mitochondria in action of artemisinin. PLoS Genet 1(3): e36.

51. Emadi A, Ross AE, Cowan KM, Fortenberry YM, Vuica-Ross $M$ (2010). A chemical genetic screen for modulators of asymmetrical 2,2'-dimeric naphthoquinones cytotoxicity in yeast. PLoS One 5(5): e10846.

52. Miramar MD, Costantini $P$, Ravagnan L, Saraiva LM, Haouzi D, Brothers G, Penninger JM, Peleato ML, Kroemer G, Susin SA (2001). NADH oxidase activity of mitochondrial apoptosis-inducing factor. J Biol Chem 276(19): 16391-16398.

53. Marshall KR, Gong M, Wodke L, Lamb JH, Jones DJ, Farmer PB, Scrutton NS, Munro AW (2005). The human apoptosis-inducing protein AMID is an oxidoreductase with a modified flavin cofactor and DNA binding activity. J Biol Chem 280(35): 30735-30740.

54. Brust D, Hamann A, Osiewacz HD (2010). Deletion of PaAif2 and PaAmid2, two genes encoding mitochondrial AIF-like oxidoreductases of Podospora anserina, leads to increased stress tolerance and lifespan extension. Curr Genet 56(3): 225-235.

55. Goncalves AP, Hall C, Kowbel DJ, Glass NL, Videira A (2014). CZT-1 Is a Novel Transcription Factor Controlling Cell Death and Natural Drug Resistance in Neurospora crassa. G3 (Bethesda) 4(6): 1091-1102.

56. Liu Z, Butow RA (1999). A transcriptional switch in the expression of yeast tricarboxylic acid cycle genes in response to a reduction or loss of respiratory function. Mol Cell Biol 19(10): 6720-6728. 